\title{
The role of two periplasmic copper- and zinc-cofactored superoxide dismutases in the virulence of Salmonella choleraesuis
}

\author{
Assunta Sansone, ${ }^{1}$ Patricia R. Watson, ${ }^{2}$ Timothy S. Wallis, ${ }^{2}$ \\ Paul R. Langford ${ }^{1}$ and J. Simon Kroll ${ }^{1}$
}

\footnotetext{
1 Molecular Infectious Diseases Group

Department of Paediatrics, Faculty of Medicine, Imperial College, St Mary's Hospital Campus, Norfolk Place, London W2 1PG, UK

2 Institute for Animal Health, Compton, Newbury, Berkshire, RG20 7NN, UK
}

Author for correspondence: J. Simon Kroll. Tel: +44 207886 6220. Fax: +44 2078866284. e-mail:s.kroll@ic.ac.uk

Periplasmic copper- and zinc-cofactored superoxide dismutases ([Cu,Zn]-SODs, SodC) of several Gram-negative pathogens can protect against superoxideradical-mediated host defences, and thus contribute to virulence. This role has been previously defined for one [Cu,Zn]-SOD in various Salmonella serovars. Following the recent discovery of a second periplasmic [Cu,Zn]-SOD in Salmonella, the effect of knockout mutations in one or both of the original sodC-1 and the new sodC-2 on the virulence of the porcine pathogen Salmonella choleraesuis is investigated here. In comparison to wild-type, while sodC mutants - whether single or double - showed no impairment in growth, they all showed equally enhanced sensitivity to superoxide and a dramatically increased sensitivity to the combination of superoxide and nitric oxide in vitro. This observation had its correlate in experimental infection both ex vivo and in vivo. Mutation of sodC significantly impaired survival of $S$. choleraesuis in interferon $\gamma$-stimulated murine macrophages compared to wild-type organisms, and all S. choleraesuis sodC mutants persisted in significantly lower numbers than wild-type in BALB/c $\left(I t y^{s}\right)$ and $\mathrm{C} 3 \mathrm{H} / \mathrm{HeN}\left(I t y^{r}\right)$ mice after experimental infection, but in no experimental system were sodC-1 sodC-2 double mutants more attenuated than either single mutant. These data suggest that both $[\mathrm{Cu}, \mathrm{Zn}]-S O D s$ are needed to protect bacterial periplasmic or membrane components. While SodC plays a role in S. choleraesuis virulence, the data presented here suggest that this is through overcoming a threshold effect, probably achieved by acquisition of sodC-1 on a bacteriophage. Loss of either sodC gene confers maximum vulnerability to superoxide on S. choleraesuis.

Keywords: Superoxide dismutase, Salmonella, periplasm, bacterial [Cu,Zn]-SOD, bacterial virulence

\section{INTRODUCTION}

Salmonella serovars responsible for disease in various mammalian hosts can proliferate as facultative intracellular pathogens, able to survive within the phagosome and/or phagolysosome of macrophages (Buchmeier \&

Abbreviations: $[\mathrm{Cu}, \mathrm{Zn}]-\mathrm{SOD}$, copper- and zinc-cofactored superoxide dismutase; DEDC, diethyldithiocarbamate; FCS, foetal calf serum; IFN $\gamma$,

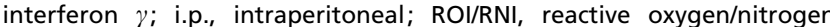
intermediate; SOD, superoxide dismutase; SPER/NO, 2,2'-hydroxy-nitrosohydrazono bis-ethanamine (spermine NONOate); $\mathrm{X}$, xanthine; XO, xanthine oxidase.
Heffron, 1989). Salmonella virulence in mice has been correlated with the net growth of Salmonella within these cells of the reticuloendothelial system (Fields et al., 1986). This suggests a capacity to resist host defences including the superoxide radical anion $\left(\mathrm{O}_{2}^{-*}\right)$ generated during the respiratory burst, nitric oxide $(\mathrm{NO})$ produced by inducible NO synthase and a range of reactive molecules formed by reaction between these two species. These include various extremely reactive oxygen and nitrogen intermediates (ROIs, RNIs) such as the hydroxyl radical and peroxynitrite that exert a potent microbicidal effect through the indiscriminate oxidative damage they cause to lipids, proteins and DNA in cell 
membranes, the periplasm and cytosol. Diverse mechanisms are beginning to be defined which contribute to the capacity of Salmonella strains to withstand exogenous ROI and RNI stress. The superoxide radical causes oxidative damage to iron-sulphur clusters, and the product of the $y g g X$ gene has been shown to exert a protective effect (Gralnick \& Downs, 2001). The microbicidal effects of RNIs derived from phagocytic cell metabolism are in part vitiated by a recently described bacterial alkyl hydroperoxide reductase (AhpC) (Chen et al., 1998), while the very production in the macrophage phagosome of these toxic species is impaired through the operation of a Salmonella pathogenicity island 2-encoded type III secretion system that mediates exclusion of NADPH oxidase assembly from the phagosome membrane (Gallois et al., 2001). Overall, the superoxide radical is a key intermediate in the production of these toxic species, and bacterial superoxide dismutases might accordingly be expected to play an important part in endowing pathogenic organisms with resistance to such host defences. Recent light has been thrown on this process with the description of the role of bacterial periplasmic copper- and zinc-cofactored superoxide dismutases ([Cu,Zn]-SODs) in the virulence of various serovars of Salmonella (Farrant et al., 1997; De Groote et al., 1997).

Superoxide dismutases (SODs) are virtually ubiquitous in bacteria, catalysing the conversion of $\mathrm{O}_{2}^{-\cdot}$ into hydrogen peroxide and oxygen (McCord \& Fridovich, 1969) in the first of a series of reactions to remove free radicals generated during the reduction of molecular oxygen. Three types of SOD are widely found in bacteria. Two are cofactored by manganese or iron and are located in the cytoplasm where they catalyse dismutation of $\mathrm{O}_{2}^{-\cdot}$ generated in the course of aerobic metabolism. A third SOD is cofactored by copper and zinc ([Cu, $\mathrm{Zn}]-\mathrm{SOD})$ and has been identified in the periplasm of a wide range of Gram-negative bacteria [Photobacterium leiognathi (Steinman, 1987); Caulobacter crescentus (Steinman, 1982); Brucella abortus (Stabel et al., 1994); Haemophilus species (Langford et al., 1992; Kroll et al., 1995); Legionella species (St John \& Steinman, 1996); Actinobacillus and Pasteurella species (Kroll et al., 1995); Escherichia coli (Benov \& Fridovich, 1994; Imlay \& Imlay, 1996); and Salmonella typhimurium, Salmonella choleraesuis and Salmonella dublin (Canvin et al., 1996; Farrant et al., 1997)]. Within the periplasm $[\mathrm{Cu}, \mathrm{Zn}]-\mathrm{SOD}$ is inaccessible to cytosolic superoxide but is suitably located to protect the organism from exogenous superoxide and the toxic action of its further reaction products with hydrogen peroxide and nitric oxide. Studies have confirmed such a function both in Salmonella and Neisseria meningitidis (Farrant et al., 1997; De Groote et al., 1997; Wilks et al., 1998).

The degree of dissimilarity (only $54 \%$ identity) between the translated Salmonella sodC sequence described by Farrant et al. (1997) and E. coli SodC, its aberrant map position with respect to the Salmonella and E. coli genomes, and its flanking regions encoding proteins resembling products of bacteriophage lambda, suggest- ed that this gene was not the orthologue of the E. coli gene and that Salmonella may have acquired it through phage-mediated transfer from an unknown donor (Farrant et al., 1997). This hypothesis has been supported by the discovery of a new Salmonella gene, sodC-2, encoding a protein $82 \%$ identical to the E. coli SodC (Fang et al., 1999). The first sodC gene is now renamed sodC-1. Here we report an investigation of the contribution that each of these genes makes to the virulence of $S$. choleraesuis. Unexpectedly, we did not find the effect to be additive, but rather, that both genes were necessary for the contribution of $[\mathrm{Cu}, \mathrm{Zn}]-\mathrm{SOD}$ to be discerned.

\section{METHODS}

Bacterial strains and culture. S. choleraesuis strain A50 is a clinical veterinary isolate (Watson et al., 2000). A $\operatorname{sodA}$ mutant of $S$. typhimurium was kindly provided by Dr Anjam Khan (University of Newcastle). E. coli S17-1 $\lambda$-pir was used as the host for conjugative transfer of plasmid DNA to Salmonella (Simon et al., 1983). Salmonella strains were made resistant to nalidixic acid by standard procedures. Bacteria were grown in Luria-Bertani (LB) medium at $37^{\circ} \mathrm{C}$ with shaking (130-180 r.p.m.). Where needed, antibiotics were added at: kanamycin, $50 \mathrm{mg} \mathrm{l}^{-1}$; nalidixic acid, $30 \mathrm{mg} \mathrm{l}^{-1}$; penicillin, $250 \mathrm{mg} \mathrm{l}^{-1}$. Bacterial strains were stored in $10 \%$ (v/v) glycerol at $-70{ }^{\circ} \mathrm{C}$ and fresh aliquots were used for each infection experiment.

Construction of sodC-2 mutants. A $344 \mathrm{bp}$ DNA fragment amplified by PCR from within the sodC-2 ORF (nt 508-851, GenBank accession AF056931) and ligated into the suicide vector pRR10( $\Delta t r f A)$ (Fang et al., 1992) was generously provided by Ferric Fang (University of Colorado). This plasmid was mobilized from E. coli S17-1 into $S$. choleraesuis A50 wild-type and sodC-1 mutant (Farrant et al., 1997). Transconjugants were selected on LB agar containing penicillin for the sodC-2 mutants or kanamycin and penicillin for the sodC-1 sodC-2 mutants. Homologous recombination of the plasmid into the Salmonella chromosome created an interruption of sodC-2, confirmed by Southern hybridization.

Extraction of bacterial proteins and detection of SOD activity. Periplasmic extracts were prepared from $100 \mathrm{ml}$ overnight cultures of $S$. choleraesuis wild-type, and sodC-1, sodC-2 and sodC-1 sodC-2 mutants by the method of Higgins \& Hardie (1983). Proteins were precipitated with ammonium sulphate $\left(650 \mathrm{~g} \mathrm{l}^{-1}\right)$, concentrated by centrifugation and stored at $-20{ }^{\circ} \mathrm{C}$ for later analysis. To detect SOD, proteins were separated in pre-cast wide range ( $\mathrm{pH} 3-10)$ IEF gels (Bio-Rad). Gels were stained to reveal SOD activity using the method of Beauchamp \& Fridovich (1971) as modified by Steinman (1985). [Cu,Zn]-SOD activity was identified by specific inhibition with $5 \mathrm{mM}$ diethyldithiocarbamate (DEDC; Sigma), a copper chelator (Benov \& Fridovich, 1994). [Fe]-SOD was identified by inhibition of its activity with hydrogen peroxide (Crapo et al., 1978).

Bacterial sensitivity to superoxide and nitric oxide generated in vitro. Sensitivity to increased cytosolic $\mathrm{O}_{2}^{-\cdot}$ flux induced by paraquat was assessed by the method of De Groote et al. (1995). Sensitivity to exogenous $\mathrm{O}_{2}^{-\cdot}$ and $\mathrm{NO}$ was assessed by exposure of suspensions $\left(10^{5}\right.$ c.f.u. $\left.\mathrm{ml}^{-1}\right)$ of stationary-phase organisms in phosphate-buffered saline $(\mathrm{pH} 7 \cdot 4)(\mathrm{PBS})$ to these radicals, generated in solution respectively from reactions 
between xanthine $(\mathrm{X})$ and xanthine oxidase (XO) (Fridovich, 1970 ) and on acidification of ice cold alkaline 2,2'-hydroxynitrosohydrazono bis-ethanamine (spermine NONOate, SPER/NO) (De Groote et al., 1997). Bacterial suspensions in these solutions were incubated with shaking at $37^{\circ} \mathrm{C}$ and aliquots were removed periodically and assayed for viable counts.

Preparation of macrophages. Porcine alveolar macrophages were isolated as previously described (Farrant et al., 1997). Macrophages were suspended in Iscove's modified Dulbecco's medium containing $10 \%(\mathrm{v} / \mathrm{v})$ foetal calf serum (FCS), $100 \mathrm{mg}$ gentamicin $\mathrm{l}^{-1}$ and $100 \mathrm{U} \mathrm{ml}^{-1}$ each of penicillin and streptomycin, and distributed into $1 \mathrm{ml}$ flat-bottomed wells of tissue culture plates to give $5 \times 10^{5}$ cells per well. Wells were incubated overnight at $37^{\circ} \mathrm{C}$ in an atmosphere of $5 \% \mathrm{CO}_{2}$ to allow cells to adhere as monolayers. Two hours before infection monolayers were washed and covered with fresh antibiotic-free medium. Murine peritoneal macrophages were isolated from BALB/c mice at 4 days after intraperitoneal (i.p.) injection of $5 \mathrm{mM}$ sodium periodate by peritoneal lavage. Macrophages were suspended in RPMI 1640 medium containing $10 \%$ FCS and $20 \mathrm{U}$ murine interferon $\gamma(\mathrm{IFN} \gamma) \mathrm{ml}^{-1}$, and distributed into $0 \cdot 1 \mathrm{ml}$ flat-bottomed wells at $5 \times 10^{4}$ cells per well. Cells were left to adhere as monolayers as before, and washed and covered with fresh medium at the start of experiments.

Infection of monolayers. Bacteria from overnight cultures were washed and resuspended in tissue culture medium at a concentration of $5 \times 10^{6}$ c.f.u. $\mathrm{ml}^{-1}$. For opsonization, bacteria were incubated with $10 \%$ normal porcine or murine serum at $37^{\circ} \mathrm{C}$ for $30 \mathrm{~min}$ on a rolling platform at 120 r.p.m. Bacteria were added to monolayers to give a verified multiplicity of infection of 5-10 bacteria per macrophage. In experiments with IFN $\gamma$-stimulated macrophages, the infected monolayers were gently centrifuged $\left(150 \mathrm{~g}\right.$ for $10 \mathrm{~min}$ at $\left.4^{\circ} \mathrm{C}\right)$. Experiments were performed in triplicate.

Bacterial uptake and killing by macrophages. Bacterial uptake and killing were assessed by a modification of the gentamicinprotection assay of Buchmeier \& Heffron (1989). Infected monolayers of IFN $\gamma$-stimulated macrophages were incubated for $15 \mathrm{~min}$, followed by a wash and further incubation with Iscove's modified Dulbecco's Medium or RPMI 1640 containing $10 \% \mathrm{v} / \mathrm{v}$ FCS and $100 \mathrm{mg}$ gentamicin $\mathrm{l}^{-1}$. After $1-24 \mathrm{~h}$ incubation, monolayers were washed twice with PBS to remove any remaining superficially adherent viable bacteria and lysed with $0 \cdot 1 \%(\mathrm{w} / \mathrm{v})$ sodium deoxycholate in PBS. To assess viable counts of phagocytosed bacteria, lysates were plated on to MacConkey agar containing $30 \mathrm{mg}$ nalidixic acid $1^{-1}$. For experiments with unstimulated macrophages, the initial incubation was extended to $1 \mathrm{~h}$.

Experimental infection of mice. Mice were purchased from Charles River Laboratories. In a first experiment, groups of five $8-10$ week-old BALB/c female mice were injected i.p. with $10^{3}$ c.f.u., and five $\mathrm{C} 3 \mathrm{H} / \mathrm{HeN}$ mice with $5 \times 10^{4}$ c.f.u. A group of sham-inoculated mice received saline only. At 4 and 5 days post-infection, respectively, when animals infected with the wild-type $S$. choleraesuis began to exhibit symptoms of systemic disease (ruffled fur, reduced activity), all the mice were killed by $\mathrm{CO}_{2}$ asphyxiation followed by cervical dislocation. Spleens and livers were removed, weighed and homogenized in $0.9 \%$ saline containing $1 \%$ Triton X-100. Viable counts were determined by plating serial dilutions on MacConkey agar containing nalidixic acid. Replica plating on appropriate selective media was carried out to exclude the theoretical possibility that mutant strains might have reverted to wild-type.

In a second experiment, groups of three 8-10 week-old BALB/c female mice were infected i.p. with 100 c.f.u. Salmonella suspended in $0.1 \mathrm{ml} 0.9 \%$ saline or saline only (sham infection). At 4 days post-infection the mice began to exhibit symptoms of systemic disease and were killed by $\mathrm{CO}_{2}$ asphyxiation. Spleens and livers were removed and number of viable organisms in the organs was determined as above.

Groups of three 8-10 week-old BALB/c female mice were infected by oral administration of $10^{6}$ c.f.u. Salmonella suspended in $0 \cdot 1 \mathrm{ml} 0 \cdot 9 \%$ saline. Sham-infected mice received saline only. At 5 days post-infection, as mice began to exhibit symptoms of systemic disease, they were killed by $\mathrm{CO}_{2}$ asphyxiation. Spleens and livers were removed and the number of viable organisms in the organs was determined as above.

\section{RESULTS}

\section{Characterization of mutants and growth characteristics}

The presence of sodC-1 and sodC-2 was established in $S$. choleraesuis by Southern blotting (Farrant et al., 1997 and further data not shown) and the set of sodC-1 and sodC-2 mutants of strain A50 were constructed as described in Methods. Strains were examined for SOD activity. Two bands of SOD activity, sensitive to DEDC inhibition and so characteristic of $[\mathrm{Cu}, \mathrm{Zn}]-\mathrm{SOD}$, were present in the wild-type but one or other band, as expected, was absent in the single mutant strains (Fig. 1). SodC-2 is more acidic (pI 5-6) than SodC-1 (pI $\sim 8$ ) and runs very close to a band of [Fe]-SOD activity, sensitive to hydrogen peroxide $\left(\mathrm{H}_{2} \mathrm{O}_{2}\right.$ sensitivity data not shown). S. choleraesuis A50 wild-type and mutant strains were grown aerobically in broth culture with shaking for $78 \mathrm{~h}$, without replenishment of the LB medium. There was no significant difference in gen- (a)

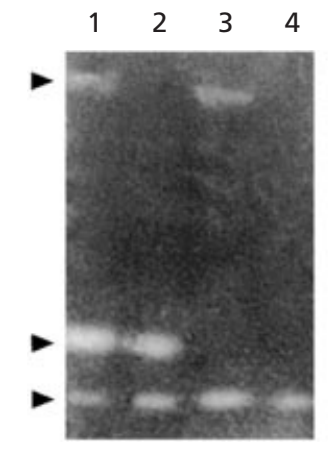

(b)

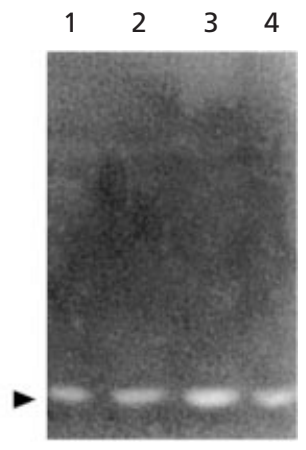

Fig. 1. Paired IEF gels stained for SOD activity (a) without and (b) with selective inhibition of [Cu,Zn]-SODs by $5 \mathrm{mM}$ DEDC. The $\mathrm{pH}$ range of the ampholytes was 10 (top) to 3 (bottom). Lanes 1-4 contain proteins extracted from (1) S. choleraesuis A50 wild-type; (2) S. choleraesuis sodC-1 mutant; (3) S. choleraesuis sodC-2 mutant; and (4) S. choleraesuis sodC-1 sodC-2 double mutant. Arrowheads mark the positions of SodC-1 (top), SodC-2 (middle) and iron-cofactored SOD (bottom). 


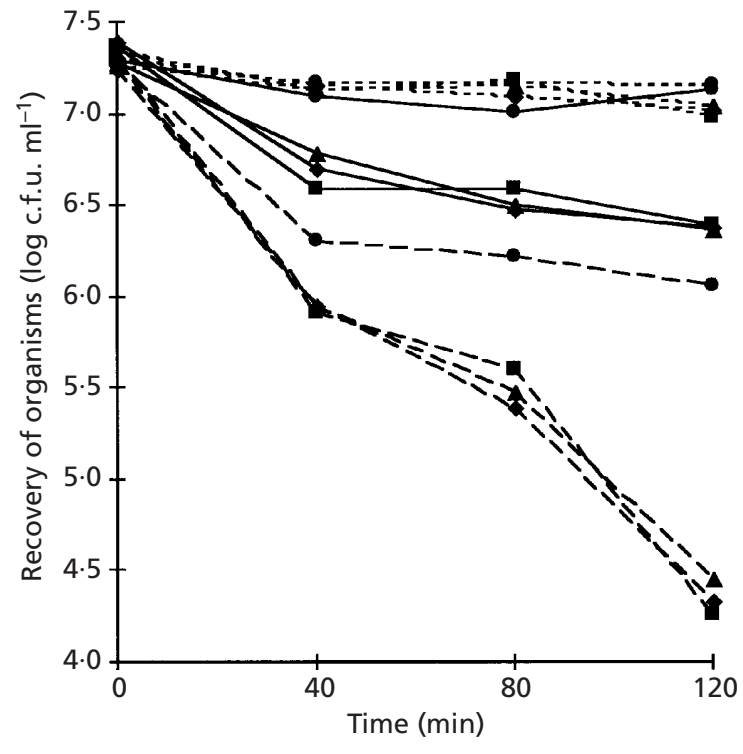

Fig. 2. Effect of sodC mutations on survival of $S$. choleraesuis on exposure to $\mathrm{O}_{2}^{-}$and $\mathrm{NO}$ generated in solution. Recovery of wild-type (O), sodC-1 ( $\mathbf{\square})$, sodC-2 ( $\bullet$ ) and double ( $\mathbf{\Delta})$ mutant strains in PBS $+X / X O$ (continuous lines), PBS + SPER/NO (dotted lines) and $\mathrm{PBS}+\mathrm{X} / \mathrm{XO}+\mathrm{SPER} / \mathrm{NO}$ (dashed lines). A representative result of the experiment (carried out in triplicate) is shown.

eration time between wild-type and mutants during exponential growth, in maximum density achieved $\left(10^{10}\right.$ c.f.u. $\mathrm{ml}^{-1}$ ) and in viability after prolonged stationary phase culture (data not shown).

\section{Effect of mutations on sensitivity to reactive oxygen and nitrogen species in vitro}

As expected, wild-type and sodC mutant strains were equally resistant to paraquat, a redox cycling reagent that penetrates the cytosol, greatly increasing superoxide production there. S. typhimurium $\Delta$ sodA (lacking cytosolic Mn-cofactored SOD) was used as the control and was shown to be more sensitive (data not shown). $S$. choleraesuis mutant strains were significantly $(P<0 \cdot 01)$ more susceptible than the wild-type to killing by extracellular superoxide generated by X/XO (Fig. 2). A dramatically increased sensitivity of the mutants was observed to the synergistic killing by $\mathrm{X} / \mathrm{XO}$ and the $\mathrm{NO}$ donor (Fig. 2). SPER/NO by itself had no antimicrobial activity against either $S$. choleraesuis wild-type or mutant strains. No additional difference in sensitivity was shown by the double mutant in any of these assays.

\section{[Cu,Zn]-SODs and interaction of Salmonella with phagocytic cells in vitro}

S. choleraesuis sodC mutant strains showed a significant increased susceptibility to killing by activated macrophages compared to the wild-type strain (Fig. 3). There was no difference in the susceptibility of the single mutants, or between either single and the double mutant.

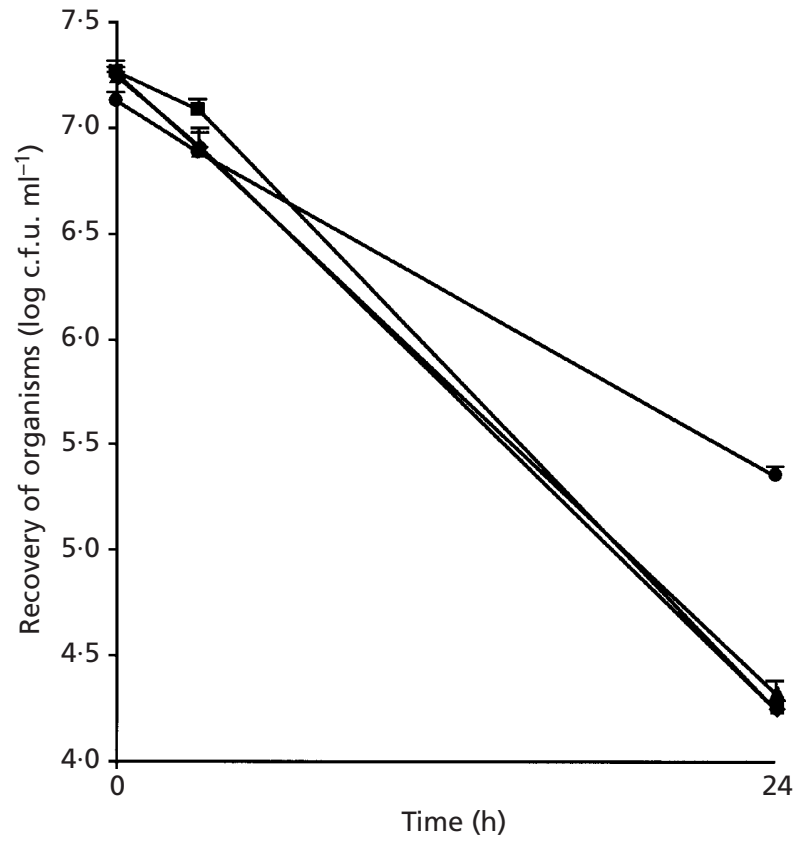

Fig. 3. Effect of sodC mutations on the persistence of $S$. choleraesuis wild-type ( $)$ ), sodC-1 ( $\square)$, sodC-2 $(\diamond)$ and double (A) mutant strains in INF $\gamma$-stimulated periodate-elicited peritoneal macrophages from BALB/c mice. Recovery of organisms from lysed cells expressed as mean (+SEM) of triplicate wells for each time point is shown.

Macrophage activation was necessary to elicit these differences. Using non-activated murine and porcine macrophages, prepared as described by Farrant et al. (1997), no differences were found between wild-type and $\operatorname{sodC}$ mutant strains in either bacterial uptake or subsequent bacterial killing, whether or not bacteria were opsonized (results not shown).

\section{[Cu,Zn]-SODs and pathogenesis of systemic disease}

After i.p. infection, at the humane end point when mice began to show signs of systemic infection, the load of organisms in spleen and liver was measured as described. Whether recovered from Ity ${ }^{\mathrm{S}}$ or from Ity ${ }^{\mathrm{r}}$ mice, the wildtype strain was recovered in highly significantly greater numbers than the sodC mutant strains (Fig. 4). However, the double mutation did not attenuate any more than either single mutation. In all cases, the strains retained the expected antibiotic resistance, showing that no reversion of the sodC genotype had occurred. A similar result was obtained using lower i.p. inocula (100 c.f.u.) of bacteria (data not shown) and with groups of mice infected orally (Fig. 5). Once again the double mutation did not have any additional effect. We have previously demonstrated that complementation of one sodC mutation $(\operatorname{sod} C-1)$ with a plasmid-borne copy of the gene restored $S$. choleraesuis to wild-type virulence in this model (Farrant et al., 1997). 

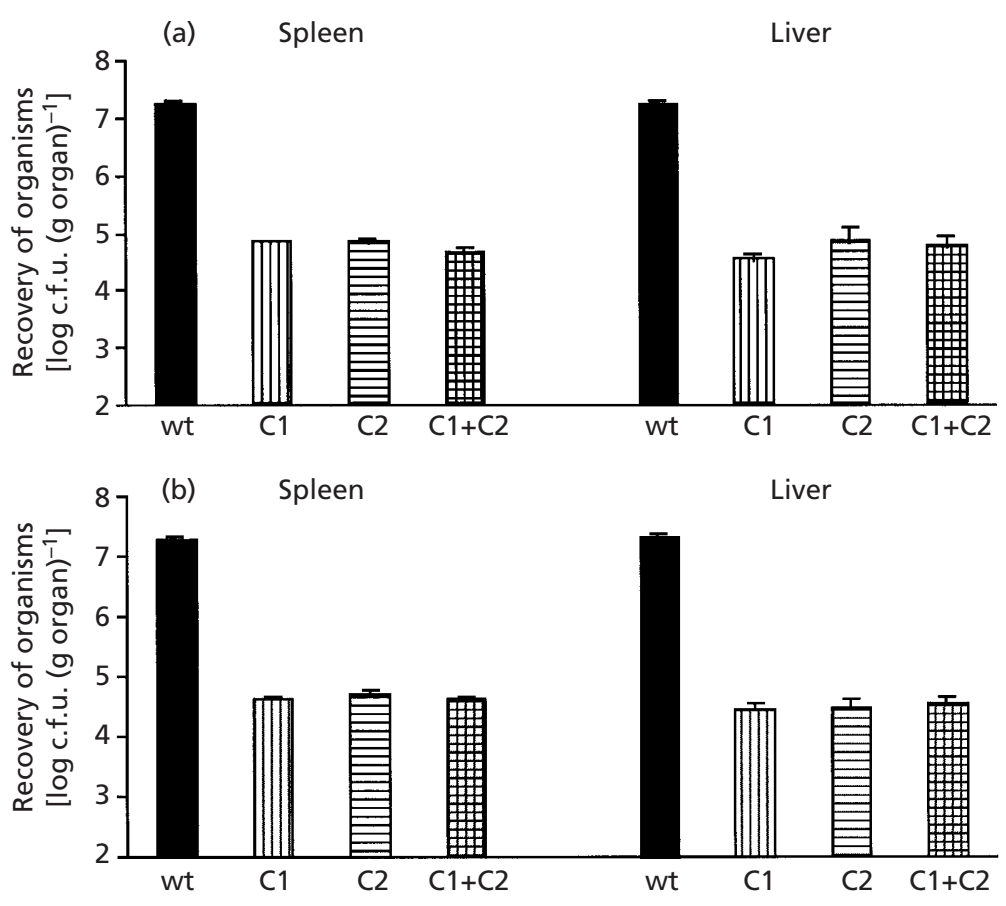

Fig. 4. Effect of sodC mutations on the virulence of $S$. choleraesuis after i.p. challenge of (a) BALB/C (4 days after challenge) and (b) $\mathrm{C} 3 \mathrm{H} / \mathrm{HeN}$ mice (5 days after challenge). Bars show the recoveries of wild-type (wt, black bars), sodC-1 (C1, vertical hatched bars), sodC-2 (C2, horizontal hatched bars) and double $(\mathrm{C} 1+\mathrm{C} 2$, crosshatched bars) mutant strains from spleen and liver. Results are expressed as c.f.u. recovered (mean +SEM) from 5 mice per bacterial strain.

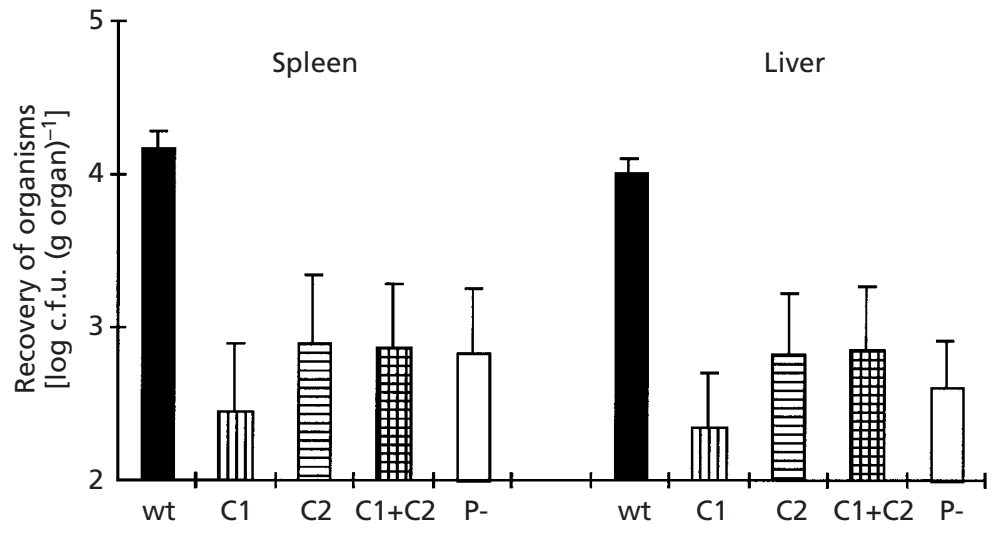

Fig. 5. Effect of sodC mutations on the virulence of $S$. choleraesuis in BALB/c mice 5 days after oral challenge. Bars show the recoveries of wild-type (wt, black bars), sodC-1 (C1, vertical hatched bars), sodC-2 (C2, horizontal hatched bars) and double $(\mathrm{C} 1+\mathrm{C} 2$, cross-hatched bars) mutant strains, and virulence plasmid-cured strain $(\mathrm{P}-$, white bars) from spleen and liver. Results are expressed as c.f.u. recovered (mean + SEM) from 3 mice per strain.

(a)

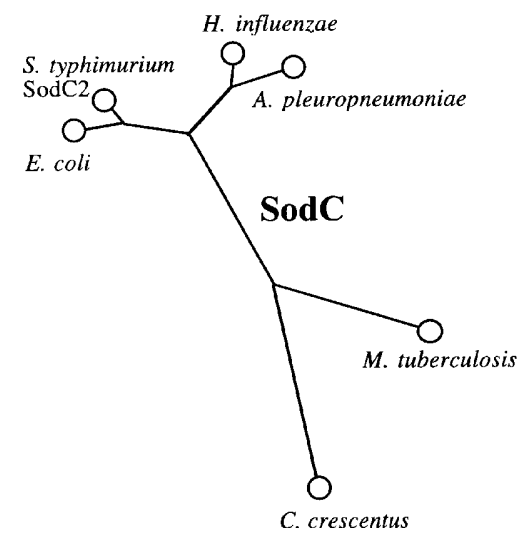

(b)

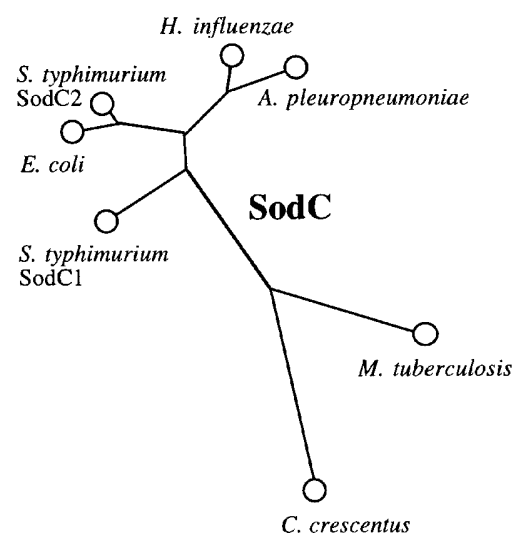

(c)

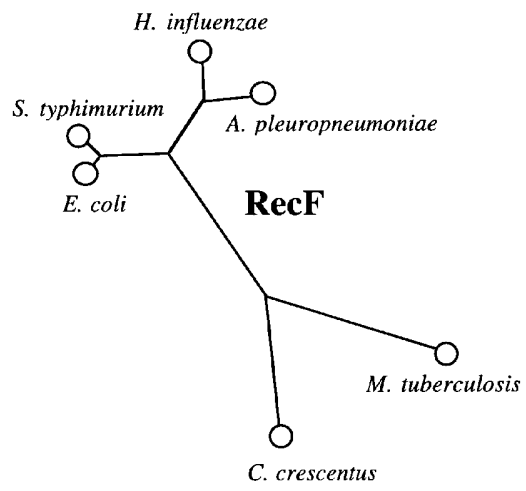

Fig. 6. Graphical representation of relationship between sequences of (a) Salmonella SodC-2 and other bacterial SodCs; (b) as (a), but including Salmonella SodC-1; (c) RecF from the same bacterial species. 


\section{Phylogenetic comparison of Salmonella SodC-1 and SodC-2 with SodC from other bacteria}

The multiple sequence comparison programme UnrootedTree (at http://cbrg.inf.ethz.ch/Server/MultAlign. html) was used to analyse graphically the relationship between the published sequences of Salmonella SodCs and SodC from the disparate species E. coli, Haemophilus influenzae, Actinobacillus pleuropneumoniae, Mycobacterium tuberculosis and C. crescentus (Fig. 6). A similar analysis can be carried out for any set of homologous proteins, and for comparison is shown for the central metabolic (DNA repair) enzyme RecF from the same bacterial species (Fig. 6c). These phylogenetic trees are drawn on the principle that the length of the path joining loci representing protein sequences is in proportion to their degree of dissimilarity. Short and long distances thus signify, respectively, similar or divergent sequences. The striking degree of similarity between the RecF and SodC trees before Salmonella SodC-1 is taken into account (Fig. 6a compared to Fig. 6c) suggests that $\operatorname{sod} C$ and $r e c F$ are not subject to different pressure of mutation in the different species analysed. Salmonella and E. coli protein sequences are generally very similar and the short distance separating the RecF sequence loci corresponds to that separating Salmonella SodC-2 and E. coli SodC. However, when the tree is reconstructed to include Salmonella SodC-1 (Fig. 6b), this branch diverges widely from both Salmonella SodC-2 and E. coli SodC.

\section{DISCUSSION}

Here we have shown that extracytoplasmic [Cu,Zn]SODs in Salmonella protect organisms against exogenously generated $\mathrm{O}_{2}^{-\cdot}$. In addition we have demonstrated the contribution of SodC- 1 and SodC- 2 to the virulence of $S$. choleraesuis in an experimental infection model, establishing [ $\mathrm{Cu}, \mathrm{Zn}]-\mathrm{SOD}$ as a determinant of Salmonella virulence, reducing the effectiveness of ROIand RNI-mediated host defence mechanisms. While the two SodCs together are associated with full virulence of $S$. choleraesuis, our data show that their contributions are not additive. Inactivation of both sodC genes leads to no further attenuation in the resistance of $S$. choleraesuis to $\mathrm{O}_{2}^{-\cdot}$ and $\mathrm{NO}$ generated in vitro, or in virulence, than is seen with either single mutant. This suggests that the contribution of $[\mathrm{Cu}, \mathrm{Zn}]-\mathrm{SOD}$ in these respects is conditional on there being a threshold level of enzyme activity. With two functional sodC genes, S. choleraesuis achieves enhanced survival under the conditions of oxidative stress encountered during experimental murine infection by the oral or parenteral route, reproduced ex vivo in experimental infection of activated macrophages. This observation finds a correlate in the observations of Battistoni et al. (2000). An E. coli strain engineered to increase [ $\mathrm{Cu}, \mathrm{Zn}]-\mathrm{SOD}$ production was found to be much more resistant to intracellular killing than the wild-type strain, while there was very little difference in survival between the wild-type and a $\operatorname{sod} C$ null mutant.
While a [Cu, $\mathrm{Zn}]-\mathrm{SOD}$ gene has by now been found in many bacterial species, the discovery of two different, functional, versions of the gene - and very recently a third, sodC-3 (Figueroa-Bossi et al., 2001) - in the one pathogen is without precedent. The gene sodC-2 in Salmonella is the true orthologue of sodC in E. coli, established by extended sequencing of the chromosomal locus in each organism. In E. coli a $7 \mathrm{~kb}$ domain contains consecutive ORFs: slyA-B1643-B1644-B1645-sodCB1647-B1648-B1649-nemA. A search with the translation of each of these genes/putative genes against the S. typhimurium genome database (at http://genome. wustl.edu/gsc/Projects/S.typhimurium/) demonstrated in each case high identity ( $>80 \%$ ) for corresponding ORFs flanking sodC-2. In contrast, sodC-1 and sodC-3 appear to have been acquired on lysogenic bacteriophages (Gifsy-2 and Fels-1 respectively) (Figueroa-Bossi \& Bossi, 1999; Figueroa-Bossi et al., 2001). A likely origin of sodC-1 from outside Salmonella is revealed by all-against-all protein sequence comparison of a set of SodC sequences. SodC-1 is as divergent in sequence from Salmonella SodC-2 and E. coli SodC as the examples of the sodC from such upper respiratory tract pathogens as H. influenzae. While an anomalously high mutation rate could theoretically be the explanation for the SodC-1 sequence divergence, the association with phage genes strongly supports the hypothesis that this gene has an exogenous origin: sodC-1 joins a growing list of virulence genes likely to have been acquired through horizontal transfer. Carriage of sodC-1 on lysogenized Gifsy-2 is a characteristic of the more virulent Salmonella serotypes, such as S. typhimurium, S. choleraesuis, S. dublin and Salmonella enteritidis. The less virulent serotypes have only sodC-2 (Fang et al., 1999). Taken with the data presented here for $S$. choleraesuis, this suggests that selection pressure may have favoured acquisition of one or even two extra $[\mathrm{Cu}, \mathrm{Zn}]-\mathrm{SOD}$ genes by Salmonella serovars adapted to survive within macrophages. A mechanism to promote sodC-1 acquisition may be proposed. Gifsy-2 elements are released upon exposure to $\mathrm{H}_{2} \mathrm{O}_{2}$, raising the intriguing possibility that infection of macrophages and exposure to reactive oxygen species generated in the respiratory burst may, through induction of a lytic cycle for Gifsy-2, potentiate transmission of the genes it carries, among them protective sodC-1, to other salmonellae (Figueroa-Bossi et al., 1999).

The proposition that horizontally acquired $[\mathrm{Cu}, \mathrm{Zn}]-$ SOD genes may make a special contribution to bacterial virulence, not conferred by endogenous $\operatorname{sod} C$, gains support from observations in the genus Neisseria. Commensal neisserial species, common colonists of the human upper respiratory and genital mucosa, do not in general have $s o d C$ genes (our unpublished results) but $N$. meningitidis, the only neisserial species regularly causing invasive, life threatening, infection, is the exception and in this pathogen [Cu, $\mathrm{Zn}]-\mathrm{SOD}$ unequivocally contributes to virulence (Wilks et al., 1998). As seen for sodC-1 in Salmonella, meningococcal sodC appears to have been acquired by horizontal transfer, in 
this case probably from co-commensal Haemophilus species (Kroll et al., 1998). In contrast, studies of other pathogens with only a single sodC gene and in which there is no evidence, for example from sequence anomaly at the locus, of horizontal acquisition of the gene, have generally yielded equivocal or negative evidence for the involvement of $[\mathrm{Cu}, \mathrm{Zn}]-\mathrm{SOD}$ in virulence $[B$. abortus (Tatum et al., 1992; Latimer et al., 1992), A. pleuropneumoniae (Sheehan et al., 2000) and M. tuberculosis (Dussurget et al., 2001; Piddington et al., 2001)].

Fang et al. (1999) have studied the contribution that SodC-1 and SodC-2 make to the virulence of $S$. typhimurium and reached a different conclusion to ours. It is not, however, possible directly to compare our findings as there are significant differences in experimental design. In a different experimental modelmuch more prolonged murine infection to a fatal end point - they observed that only the sodC- 1 sodC-2 strain was attenuated in lethality for Ity ${ }^{\mathrm{S}}$ mice (a different strain to that used here). In an Ity ${ }^{\mathrm{r}}$ strain (the same as that used here), all sodC mutants, single and double, were slower to kill mice than the wild-type. Although the result reported for the double mutant suggested greater attenuation, the reproducibility of this single observation cannot be assessed with the data available. It may be that the contrast reflects differences in the pathogenesis of S. typhimurium and S. choleraesuis in mice. Despite being highly virulent for pigs (Watson et al., 2000), S. choleraesuis A50 is significantly less virulent for mice than S. typhimurium and S. dublin (Farrant et al., 1997).

In conclusion, in $S$. choleraesuis it seems clear that the acquisition of an additional sodC gene on a lysogenic bacteriophage has effectively led to a gain of function (plausibly through increased total activity) for [Cu, $\mathrm{Zn}]-$ SOD, contributing to virulence. Until now the contribution that sodC makes to bacterial virulence has seemed wholly capricious - clearly contributing in some organisms, but not at all in others. From this work we would tentatively suggest that it is in particular in those organisms in which there is evidence of horizontal acquisition of $\operatorname{sod} C$ - by bacteriophage or plasmid transfer, or through transformation with chromosomal DNA - that evidence may be found of a virulenceassociated gain of function, the capacity to neutralize host defences that depend on the generation of superoxide and its further reaction products.

\section{ACKNOWLEDGEMENTS}

The work described here was supported in part by grants to J.S.K. from the Meningitis Research Foundation and the Wellcome Trust.

\section{REFERENCES}

Battistoni, A., Pacello, F., Folcarelli, S. \& 7 other authors (2000). Increased expression of periplasmic $\mathrm{Cu}, \mathrm{Zn}$ superoxide dismutase enhances survival of Escherichia coli invasive strains within nonphagocytic cells. Infect Immun 68, 30-37.
Beauchamp, C. O. \& Fridovich, I. (1971). Superoxide dismutase: improved assays and an assay applicable to acrylamide gels. Anal Biochem 44, 276-287.

Benov, L. T. \& Fridovich, I. (1994). Escherichia coli expresses a copper- and zinc-containing superoxide dismutase. J Biol Chem 269, 25310-25314.

Buchmeier, N. A. \& Heffron, F. (1989). Intracellular survival of wild-type Salmonella typhimurium and macrophage-sensitive mutants in diverse populations of macrophages. Infect Immun 57, $1-7$.

Canvin, J., Langford, P. R., Wilks, K. E. \& Kroll, J. S. (1996). Identification of $\operatorname{sod} \mathrm{C}$ encoding periplasmic $[\mathrm{Cu}, \mathrm{Zn}]$-superoxide dismutase in Salmonella. FEMS Microbiol Lett 136, 215-220.

Chen, L., Xie, Q. W. \& Nathan, C. (1998). Alkyl hydroperoxide reductase subunit $\mathrm{C}(\mathrm{AhpC})$ protects bacterial and human cells against reactive nitrogen intermediates. Mol Cell 1, 795-805.

Crapo, J. D., McCord, J. M. \& Fridovich, I. (1978). Preparation and assay of superoxide dismutase. Methods Enzymol 53, 382-393.

De Groote, M. A., Granger, D., Xu, Y., Campbell, G., Prince, R. \& Fang, F. C. (1995). Genetic and redox determinants of nitric oxide cytotoxicity in a Salmonella typhimurium model. Proc Natl Acad Sci U S A 92, 6399-6403.

De Groote, M. A., Achsner, U. A., Michael, U. S. \& 7 other authors (1997). Periplasmic superoxide dismutase protects Salmonella from products of phagocyte NADPH-oxidase and nitric oxide synthase. Proc Natl Acad Sci US A 94, 13997-14001.

Dussurget, O., Stewart, G., Neyrolles, O., Pescher, P., Young, D. \& Marchal, G. (2001). Role of Mycobacterium tuberculosis copperzinc superoxide dismutase. Infect Immun 69, 529-533.

Fang, F. C., Libby, S. J., Buchmeier, N. A., Loewen, P. C., Switala, J., Harwood, J. \& Guiney, D. G. (1992). The alternative sigma factor katF (rpoS) regulates Salmonella virulence. Proc Natl Acad Sci U S A 89, 11978-11982.

Fang, F. C., De Groote, M. A., Foster, J. W. \& 8 other authors (1999). Virulent Salmonella typhimurium has two periplasmic Cu,Zn-superoxide dismutases. Proc Natl Acad Sci USA 96, 7502-7507.

Farrant, J. L., Sansone, A., Canvin, J. R., Pallen, M. J., Langford, P. R., Wallis, T. S., Dougan, G. \& Kroll, J. S. (1997). Bacterial copper-and zinc-cofactored superoxide dismutase contributes to the pathogenesis of systemic salmonellosis. Mol Microbiol 25, 785-796.

Fields, P. I., Groisman, E. A. \& Heffron, F. (1989). A Salmonella locus that controls resistance to microbicidal proteins from phagocytic cells. Science 243, 1059-1062.

Figueroa-Bossi, N. \& Bossi, L. (1999). Inducible prophages contribute to Salmonella virulence in mice. Mol Microbiol 33, 167-176.

Figueroa-Bossi, N., Uzzau, S., Maloriol, D. \& Bossi, L. (2001). Variable assortment of prophages provides a transferable repertoire of pathogenic determinants in Salmonella. Mol Microbiol 39, 260-271.

Fridovich, I. (1970). Quantitative aspects of the production of superoxide anion radical by milk xanthine oxidase. $J$ Biol Chem 245, 4053-4057.

Gallois, A., Klein, J. R., Allen, L. A., Jones, B. D. \& Nauseef, W. M. (2001). Salmonella pathogenicity island 2-encoded type III secretion system mediates exclusion of NADPH oxidase assembly from the phagosomal membrane. J Immunol 166, 5741-5748.

Gralnick, J. \& Downs, D. (2001). Protection from superoxide damage associated with an increased level of the YggX protein in Salmonella enterica. Proc Natl Acad Sci U S A 98, 8030-8035. 
Higgins, C. F. \& Hardie, M. M. (1983). Periplasmic protein associated with the oligopeptide permeases of Salmonella typhimurium and Escherichia coli. J Bacteriol 155, 1434-1438.

Imlay, K. R. C. \& Imlay, J. A. (1996). Cloning and analysis of sodC, encoding copper, zinc superoxide dismutase of Escherichia coli. J Bacteriol 178, 2564-2571.

Kroll, J. S., Langford, P. R., Wilks, K. E. \& Keil, A. D. (1995). Bacterial $[\mathrm{Cu}, \mathrm{Zn}]$-superoxide dismutase: phylogenetically distinct from the eukaryotic enzyme, and not so rare after all! Microbiology 141, 2271-2279.

Kroll, J. S., Wilks, K. E., Farrant, J. L. \& Langford, P. R. (1998). Natural genetic exchange between Haemophilus and Neisseria: intergenic transfer of chromosomal genes between major human pathogens. Proc Natl Acad Sci US A 95, 12381-12385.

Langford, P. R., Loynds, B. M. \& Kroll, J. S. (1992). Copper-zinc superoxide dismutase in Haemophilus species. J Gen Microbiol 138, 517-522.

Latimer, E., Simmers, J., Sriranganathan, N., Roop, I. R. M., Schurig, G. G. \& Boyle, S. M. (1992). Brucella abortus deficient in copper/zinc superoxide dismutase is virulent in BALB/C mice. Microb Pathog 12, 105-113.

McCord, J. M. \& Fridovich, I. (1969). Superoxide dismutase: an enzymatic function for erythrocuprein (hemocuprein). J Biol Chem 244, 6049-6055.

Piddington, D. L., Fang, F. C., Laessig, T., Cooper, A. M., Orme, I. M. \& Buchmeier, N. A. (2001). Cu, Zn superoxide dismutase of Mycobacterium tuberculosis contributes to survival in activated macrophages that are generating an oxidative burst. Infect Immun 69, 4980-4987.

Sheehan, B. J., Langford, P. R., Rycroft, A. N. \& Kroll, J. S. (2000). $[\mathrm{Cu}, \mathrm{Zn}]-$ Superoxide dismutase mutants of the swine pathogen Actinobacillus pleuropneumoniae are unattenuated in infections of the natural host. Infect Immun 68, 4778-4781.
Simon, R., Priefer, U. \& Puhler, A. (1983). A broad host range mobilization system for in vivo genetic engineering in Gram negative bacteria. Bio/Technology 1, 784-791.

Stabel, T. J., Sha, Z. \& Mayfield, J. E. (1994). Periplasmic location of Brucella abortus $\mathrm{Cu} / \mathrm{Zn}$ superoxide dismutase. Vet Microbiol 38, 307-314.

Steinman, H. M. (1982). Copper-zinc superoxide dismutase from Caulobacter crescentus CB15: a novel bacteriocuprein form of the enzyme. J Biol Chem 257, 10283-10293.

Steinman, H. M. (1985). Bacteriocuprein superoxide dismutases in pseudomonads. J Bacteriol 162, 1255-1260.

Steinman, H. M. (1987). Bacteriocuprein superoxide dismutase of Photobacterium leiognathi: isolation and sequence of the gene and evidence for a precursor form. J Biol Chem 262, 1882-1887.

St John, G. \& Steinman, H. M. (1996). Periplasmic copper-zinc superoxide dismutase of Legionella pneumophila: role in stationary phase survival. J Bacteriol 178, 1578-1584.

Tatum, F. M., Detilleux, P. G., Sacks, J. M. \& Halling, S. M. (1992). Construction of $\mathrm{Cu}-\mathrm{Zn}$ superoxide dismutase deletion mutants of Brucella abortus: analysis of survival in vitro in epithelial and phagocytic cells and in vivo in mice. Infect Immun 60, 2863-2869.

Watson, P. R., Paulin, S. M., Jones, P. W. \& Wallis, T. S. (2000). Interaction of Salmonella serotypes with porcine macrophages in vitro does not correlate with virulence. Microbiology 146, 1639-1649.

Wilks, K. E., Dunn, K. L. R., Farrant, J. L., Reddin, K. M., Gorringe, A. R., Langford, P. R. \& Kroll, J. S. (1998). Periplasmic superoxide dismutase in meningococcal pathogenicity. Infect Immun 66, 213-217.

Received 9 August 2001; revised 22 October 2001; accepted 15 November 2001. 